\title{
Noise-enabled species recovery in the aftermath of a tipping point
}

\author{
Yu Meng, ${ }^{1,2}$ Junjie Jiang, ${ }^{2}$ Celso Grebogi, ${ }^{1}$ and Ying-Cheng Lai $\odot^{2,3, *}$ \\ ${ }^{1}$ Institute for Complex Systems and Mathematical Biology, School of Natural and Computing Sciences, \\ King's College, University of Aberdeen, Aberdeen AB24 3UE, United Kingdom \\ ${ }^{2}$ School of Electrical, Computer and Energy Engineering, Arizona State University, Tempe, Arizona 85287, USA \\ ${ }^{3}$ Department of Physics, Arizona State University, Tempe, Arizona 85287, USA
}

(Received 14 June 2019; revised manuscript received 3 December 2019; published 10 January 2020)

\begin{abstract}
The beneficial role of noise in promoting species coexistence and preventing extinction has been recognized in theoretical ecology, but previous studies were mostly concerned with low-dimensional systems. We investigate the interplay between noise and nonlinear dynamics in real-world complex mutualistic networks with a focus on species recovery in the aftermath of a tipping point. Particularly, as a critical parameter such as the mutualistic interaction strength passes through a tipping point, the system collapses and approaches an extinction state through a dramatic reduction in the species populations to near-zero values. We demonstrate the striking effect of noise: when the direction of parameter change is reversed through the tipping point, noise enables species recovery which otherwise would not be possible. We uncover an algebraic scaling law between the noise amplitude and the parameter distance from the tipping point to the recovery point and provide a physical understanding through analyzing the nonlinear dynamics based on an effective, reduced-dimension model. Noise, in the form of small population fluctuations, can thus play a positive role in protecting high-dimensional, complex ecological networks.
\end{abstract}

DOI: 10.1103/PhysRevE.101.012206

\section{INTRODUCTION}

Ecological systems in the real world, in addition to being fundamentally nonlinear [1-3], are constantly subject to various random forces, rendering relevant and important studies of the effects of noise on ecological phenomena predicted in the purely deterministic framework [4-13]. For example, the benefits of noise to the health of low-dimensional, patch type of ecological systems were previously recognized $[7,8]$ where, in a spatial environment, inferior but rapidly moving species can coexist with superior but relatively stationary species, provided that there are spatiotemporal variations in the fitness. A seminal theoretical work on a competition model of two species, one inferior and another superior, in a two-patch environment demonstrated that deterministic chaotic dynamics can provide the required random or stochastic spatiotemporal variations in fitness [14]. With two populations in two patches, the model is a four-dimensional discrete-time nonlinear map. The dynamical mechanism underlying the coexistence was later found to be desynchronization and intermittency $[15,16]$ : when the population evolution of the inferior species synchronizes with that of the superior species, the abundance of the former tends to decrease. In fact, long-term synchronization can make the inferior species extinct. However, due to asymmetry and chaos, desynchronization bursts occur in an intermittent fashion, effectively preventing the population of the inferior species from collapsing. For this low-dimensional system, the presence of noise was demonstrated to be beneficial to coexistence, and a stochastic-resonance type of phenomenon was uncovered in which an optimal level of

\footnotetext{
*ying-cheng.lai@asu.edu
}

noise can significantly enhance the coexistence and thereby promote species diversity $[7,8]$.

The concept of stochastic resonance was originally proposed [17] to explain the Quaternary glacial problem. Generally, it is a phenomenon in which the presence of internal or external noise in a nonlinear system can enhance the response of the system output [17-28]. The paradigmatic setting to demonstrate stochastic resonance is a bistable system, which occurs when a periodic force is applied in the presence of a large broadband random force (e.g., noise). The system response is driven by a combination of the two forces of competition and collaboration, switching the system between two stable states. In mutualistic systems, additive white Gaussian noise constantly stimulates the system in the extinction state to drive it to a normal state.

Real-world ecological systems typically involve a large number of species and thus are high-dimensional, whose description often requires, e.g., hundreds of coupled nonlinear differential equations. In view of the demonstrated positive role of noise in low-dimensional systems, two pertinent questions arise: (1) Can the benefits extend to high-dimensional ecosystems? and (2) If the answer is affirmative, in what quantitative way? Generally, this is a difficult problem because of the scarcity of reasonably detailed mathematical models and the difficulties of analyzing and even simulating such models in the presence of noise. However, we find one class of recently developed ecological models: complex mutualistic networks [29-37] for which a generic, detailed mathematical description is available. To take advantage of this model and to investigate the effects of noise in a concrete way, we focus on the problem of species recovery in the aftermath of a tipping point transition. 
Mutualism is a close relationship between organisms of two different species in which both species benefit from the interactions [38,39]. Mutualistic networks are widely found in ecosystems. There are two typical relationships of mutualistic interactions: service-resource and service-service relationships. Typical species in the service-resource relationship are pollinators and plants, such as bees and plants, where plants provide the bees with pollen, guaranteeing their survivability, and the pollen stuck in the bee's hairy body pollinates the next plant. In a service-service relationship, species provide service to each other, such as clown fish and anemone, where anemones afford shelter to clown fish to protect them from their predators and, because of the presence of clown fish, anemones are protected from the butterfly fish.

Nonlinear ecological systems can exhibit a tipping point at which a transition from a normal to a catastrophic state occurs when a system parameter passes through a critical point $[32,36,40-53]$. Complex mutualistic networks of pollinators and plants present a paradigmatic class of such systems $[32,36]$. The gradual deterioration of the environment as a result of human activities can cause some system parameters to drift. Depending on the specific parameter, there can be distinct scenarios of system recovery in the aftermath of a tipping point transition [54]. For example, a tipping point can occur when the mutualistic interaction parameter is reduced through a critical point, below which the species abundances become near zero, driving the system effectively into an extinction state. In this case, if the environment is improved as characterized by an increase in the mutualistic parameter back through the tipping point, without external influences the system will not be able to recover even when the parameter value has been restored into the originally healthy regime of coexistence.

The main point of this paper is that, in the aftermath of a tipping point transition, if random noises in the form of stochastic fluctuations in the species abundances are present, then species recovery will be possible. In particular, let $\gamma>0$ be the normalized mutualistic interaction strength and $\gamma_{t p}$ be its critical value at the tipping point transition, as shown schematically in Fig. 1. For $\gamma<\gamma_{t p}$, both the pollinator and plant species are in the extinction state in that their abundances are near zero. Intuitively, for species recovery to occur, it is necessary only to increase the value of $\gamma$ beyond $\gamma_{t p}$. However, since the network system is already in the extinction state, recovery will not be possible simply by strengthening the mutualistic interaction beyond the tipping point value. We find that, when random noise is present, species recovery is possible when the value of $\gamma$ exceeds $\gamma_{t p}$. Let $\sigma$ be the noise amplitude and $\gamma_{c}$ be the recovery point that depends on $\sigma$ : $\gamma_{c}=\gamma_{c}(\sigma)$. Furthermore, let $\Delta \gamma(\sigma) \equiv \gamma_{c}(\sigma)-\gamma_{t p}$. Qualitatively, a more significantly improved environment means a larger value of the mutualistic interaction strength. Likewise, a small value of $\Delta \gamma_{c}(\sigma)$ means that species recovery can be achieved even without substantial and dramatic improvement in the environment, which is desired. The quantitative result of this paper is the following algebraic scaling law between $\Delta \gamma(\sigma)$ and $\sigma$ :

$$
\Delta \gamma(\sigma) \sim \sigma^{-p},
$$

where $p>0$ is the algebraic scaling exponent. (Numerically, we find that, for real-world mutualistic networks, the value of

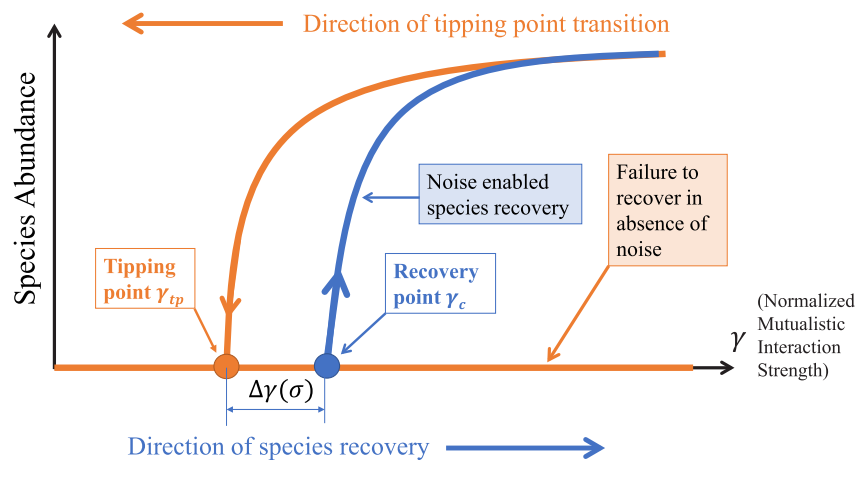

FIG. 1. A schematic illustration of tipping point transition and species recovery in a mutualistic network. We illustrate the behavior of the abundance of a typical species in the network versus the normalized strength $\gamma$ of mutualistic interaction. A tipping point transition occurs as the value of $\gamma$ is decreased through a critical value $\gamma_{t p}$, at which the species abundance decreases to a near-zero value. Recovery in the aftermath of the tipping point means increasing $\gamma$ from a value less than $\gamma_{t p}$ to some value above it. In the absence of noise, recovery is not possible, as stipulated by the fundamental nonlinear dynamics responsible for the tipping point transition (see Fig. 10 below and explanations). When noise of sufficient amplitude $\sigma$ is present, species recovery in a finite time can occur at the point $\gamma_{c}$ that depends on $\sigma$. The main result of this paper is scaling law (1), which governs how the parameter difference $\Delta \gamma \equiv \gamma_{c}-\gamma_{t p}$ scales with the noise amplitude.

the exponent is about one.) The significance of the scaling law (1) is that the value of $\Delta \gamma_{c}(\sigma)$ can be reduced by increasing the noise amplitude, making recovery more effective. We uncover the scaling law (1) through simulations of a detailed population model of pollinator and plant species for a number of high-dimensional, empirical mutualistic networks. We then obtain a physical understanding of the scaling law through a nonlinear dynamics-based argument and insights from a reduced, two-dimensional model [36]. The analysis also predicts the value of the algebraic scaling exponent $p$. Our results establish the beneficial role of noise in highdimensional ecological systems in a quantitative way, with implications to ecosystem management, conservation, and biological control. More broadly, since tipping point is a general phenomenon in nature [55], our work provides useful insights into the significant problems of mitigating tipping point transitions and recovering from the aftermath in a variety of natural and engineering systems through exploiting random noise or controlled stochastic perturbations.

\section{MODEL OF COMPLEX MUTUALISTIC NETWORKS}

Mathematically, a pollinator-plant mutualistic network can be described [29-36] by the Holling type of dynamics [56,57]. When the network is subject to independent Gaussian white noises, the mathematical equations governing the evolution of species abundances are

$$
\begin{aligned}
\frac{d X_{i}}{d t}= & \alpha_{i}^{(X)} X_{i}-\sum_{j=1}^{S_{X}} \beta_{i j}^{(X)} X_{i} X_{j}+\frac{\sum_{k=1}^{S_{Y}} \gamma_{i k}^{(X)} Y_{k}}{1+h \sum_{k=1}^{S_{Y}} \gamma_{i k}^{(X)} Y_{k}} X_{i} \\
& +\mu_{X}+\eta_{i}(t)
\end{aligned}
$$




$$
\begin{aligned}
\frac{d Y_{i}}{d t}= & \alpha_{i}^{(Y)} Y_{i}-\sum_{j=1}^{S_{Y}} \beta_{i j}^{(Y)} Y_{i} Y_{j} \\
& +\frac{\sum_{k=1}^{S_{X}} \gamma_{i k}^{(Y)} X_{k}}{1+h \sum_{k=1}^{S_{X}} \gamma_{i k}^{(Y)} X_{k}} Y_{i}+\mu_{Y}+\xi_{i}(t),
\end{aligned}
$$

where $X_{i}$ and $Y_{i}$ are the abundances of the $i$ th pollinator and $i$ th plant, $S_{X}$ and $S_{Y}$ are the numbers of pollinator and plant species, respectively, $\alpha_{i}^{(X)}$ and $\alpha_{i}^{(Y)}$ are the intrinsic growth rate in the absence of intraspecific competition and any mutualistic effect, $\beta_{i i}$ and $\beta_{i j}(i \neq j)$ are parameters characterizing intraspecific and interspecific competition, respectively, and the parameters $\mu_{X} \gtrsim 0$ and $\mu_{Y} \gtrsim 0$ characterize species migration. For the pollinator-plant system, intraspecific competition is typically stronger than interspecific competition [32,33]: $\beta_{i i} \gg \beta_{i j}$. The saturation effect is quantified by the half-saturation constant $h$. Saturation describes the situation where the beneficial effect of the mutualistic interactions on the population growth saturates when the mutualistic partners have a high abundance. It is characterized by the half-saturation density of Holling type-II functional response. The key parameters $\gamma_{i k}^{(X)}$ and $\gamma_{i k}^{(Y)}$ are the strength of the mutualistic interaction, with $\gamma_{i k}^{(X)}=0$ and $\gamma_{i k}^{(Y)}=0$ corresponding to the case of absence of mutualistic interactions. In general, the mutualistic interaction strength depends on the degree of the node as [36]

$$
\gamma_{i j}=a_{i j} \frac{\gamma}{\left(K_{i}\right)^{\rho}},
$$

where $\gamma$ is the normalized strength and $a_{i j}$ are the elements of the network adjacency matrix: $a_{i j}=1$ if there is an interaction between pollinator $i$ and plant $j$; otherwise $a_{i j}=0$. The parameter $K_{i}$ is the number of mutualistic links associated with species $i$, and $\rho$ determines the strength of the trade-off between the interaction strength and the number of interactions. If there is no trade-off (i.e., $\rho=0$ ), the network topology will have no effect on the strength of the mutualistic interactions. In contrast, a full trade-off $(\rho=1)$ means that the interaction strength is weighed by the nodal degree so the network topology will affect the species gain from the interactions. The terms that involve the mutualistic interactions for each species can then be written as [36]

$$
\begin{aligned}
& \sum_{j=1}^{S_{Y}} \gamma_{i j}^{(X)} Y_{j}=\sum_{j=1}^{S_{Y}} \frac{\gamma}{\left(K_{i}^{(X)}\right)^{\rho}} a_{i j} Y_{j}, \\
& \sum_{j=1}^{S_{X}} \gamma_{i j}^{(Y)} X_{j}=\sum_{j=1}^{S_{X}} \frac{\gamma}{\left(K_{i}^{(Y)}\right)^{\rho}} a_{i j} X_{j} .
\end{aligned}
$$

In Eqs. (2) and (3), $\eta_{i}(t)$ and $\xi_{i}(t)$ are independent Gaussian white noise with the following statistical properties:

$$
\begin{aligned}
\left\langle\eta_{i}(t)\right\rangle & =0, \\
\left\langle\eta_{i}(t) \eta_{i}(t)^{\prime}\right\rangle & =2 \sigma^{2} \delta\left(t-t^{\prime}\right), \\
\left\langle\xi_{i}(t)\right\rangle & =0, \\
\left\langle\xi_{i}(t) \xi_{i}(t)^{\prime}\right\rangle & =2 \sigma^{2} \delta\left(t-t^{\prime}\right),
\end{aligned}
$$

where $\sigma$ is the noise amplitude and $\delta(t)$ is the $\delta$ function. The phase space dimension $S_{X}+S_{Y}$ of the mutualistic networked system as described by Eqs. (2) and (3) is typically high, making a mathematical analysis difficult. However, an effective, two-dimensional reduced model is available [36], which captures the essential dynamics associated with a tipping point transition. The reduced model subject to noise can be written as

$$
\begin{aligned}
& \frac{d x}{d t}=\alpha x-\beta x^{2}+\frac{\left\langle\gamma_{x}\right\rangle y}{1+h\left\langle\gamma_{x}\right\rangle y} x+\mu+\eta(t), \\
& \frac{d y}{d t}=\alpha y-\beta y^{2}+\frac{\left\langle\gamma_{y}\right\rangle x}{1+h\left\langle\gamma_{y}\right\rangle x} y+\mu+\xi(t),
\end{aligned}
$$

where $x$ and $y$ are the effective or average abundances of the pollinator and plant species, respectively, $\alpha$ is the effective growth rate for the reduced system, $\beta$ accounts for the combined effects of intraspecific and interspecific competition, and $\mu$ characterizes the migration effect of the species. The key parameters are the two effective mutualistic interaction strength $\left\langle\gamma_{x}\right\rangle$ and $\left\langle\gamma_{y}\right\rangle$, which are obtained through properly weighed averages of the strength $\gamma_{i k}^{(X)}$ and $\gamma_{i k}^{(Y)}$ in the original empirical network [36].

For both the full and reduced models, we use the standard, second-order algorithm for solving stochastic differential equations [58] to investigate the effect of noise on system recovery in the aftermath of a tipping point transition.

It is worth clarifying the difference between the ecological systems, the modeled networks and the models used to describe the system's dynamics. In this regard, the purpose of this paper is to develop a mathematical and computational framework to study real-world empirical bipartite mutualistic networks of pollinators and plants with a particular focus on the effect of noise on the recovery from an extinction state to a normal abundance state. In a bipartite network, the nodes are divided into two disjoint sets in which every link connects a node in one set to a node in the other. The bipartite mutualistic networks that we study are an idealized but effective model of the natural system, and, of course, it cannot represent completely a real empirical system. For instance, mutualistic interactions differ among different pollinator-plant combinations. This difference is mainly manifested in the following aspects [59]: the pollinator abundances, the differences in a pollinator's preference for plants, and whether a pollinator can visit plants of a given species as well as the distance of movement for the visiting process $[60,61]$. The first aspect is the most important because it is indicative of the pollinator's effectiveness to pollinate large abundance of plants. The other two aspects involve the characteristics of the pollinators. One could get quantitative information about the characteristics of every pollinator based on the mutualistic links [62] in the empirical networks. However, the pollination ability of a give pollinator is complicated. Not only do the pollination abilities of different pollinator species differ, but the ability of the same pollinator species is time and state dependent. Consequently, measuring the actual pollinator characteristics is difficult, and its inclusion in the model is not straightforward [63]. Our Eqs. (2) and (3) are the proper model to investigate the pollinator-plant bipartite mutualistic network including stochastic effects. The pollinator-plant network is best described by Holling type-II dynamics [29-36,56,57]. 


\section{NOISE-ENABLED SPECIES RECOVERY AND SCALING LAW}

\section{A. Numerical demonstration of noise-enabled species recovery and scaling law}

\section{Empirical mutualistic networks}

We study in detail four real-world mutualistic networks derived from empirical data collected from four different geographic regions [64]: (1) network A $\left(S_{X}=61\right.$ and $S_{Y}=17$ with the number of mutualistic links $L=146$ ) from Hicking, Norfolk, United Kingdom [65], (2) network B $\left(S_{X}=38\right.$, $S_{Y}=11$, and $L=106$ ) from Tenerife, Canary Islands [66], (3) network C $\left(S_{X}=44, S_{Y}=13\right.$, and $\left.L=143\right)$ from North Carolina, USA [67], and (4) network D $\left(S_{X}=42, S_{Y}=8\right.$, and $L=79$ ) from Hestehaven, Denmark [68]. A schematic illustration of one of the networks is presented in Fig. 2.

We numerically demonstrate the phenomenon of noiseenabled species recovery and the algebraic scaling law (1). We choose the range of noise to be $\left[10^{-4}, 10^{-1}\right]$. In the aftermath of a tipping point transition, i.e., $\gamma<\gamma_{t p}$, the abundances of all species approach some near-zero values. In this case, when one attempts to recover the species by strengthening the mutualistic interactions (e.g., through significant improvement of the environment) so that the value of $\gamma$ becomes larger than $\gamma_{t p}$, without noise the network remains in the extinction state with near-zero abundance values determined by the small migration effect quantified by the near-zero migration parameters $\mu_{X}$ and $\mu_{Y}$. However, with noise the species recovery can occur as the value of $\gamma$ exceeds $\gamma_{t p}$, as shown in Fig. 3, effectively a bifurcation diagram for both the full network and the reduced model. Representative time series of the species abundances associated with the recovery process are shown in Fig. 4. For the particular value of the noise amplitude used $(\sigma=0.1)$, recovery occurs in relatively short time (comparing with the total simulation time $T=400$ ).

The model contains a large number of parameters. We choose to vary the most sensitive parameters that best highlight the dynamical behavior of the empirical network for the recovery process. The ones with fixed values are rather insensitive, not changing the scaling law and the results when varied. For instance, we have changed the parameter $h$, but its value does not affect the final result and the algebraic scaling law. The value of $\beta$ has no effect on the scaling law either. We choose five different negative values of $\alpha$ to present the scaling law. The abundances of all species do recover, as shown in Figs. 3 and 4, where the term "recovery" is used to describe the situation where all the species must recover from an extinction state. The critical value of $\gamma$ is one that leads the abundances to recover to the values in a normal state. Figure 3 shows that, with the increase of $\gamma$, the abundances of all the species will increase and exceed the values at the tipping point. The light red and light blue curves represent all the species of pollinators and plants in a full network, respectively. The dark red and dark blue curves are the average abundances of all the pollinators and plants in the network, respectively. Figure 4 demonstrates that the abundances will recover by noise perturbation. Moreover, when the unstable steady state is overcome, the abundances will escape from the low-abundance stable state (extinction state) to the high-abundance stable state (normal state) and
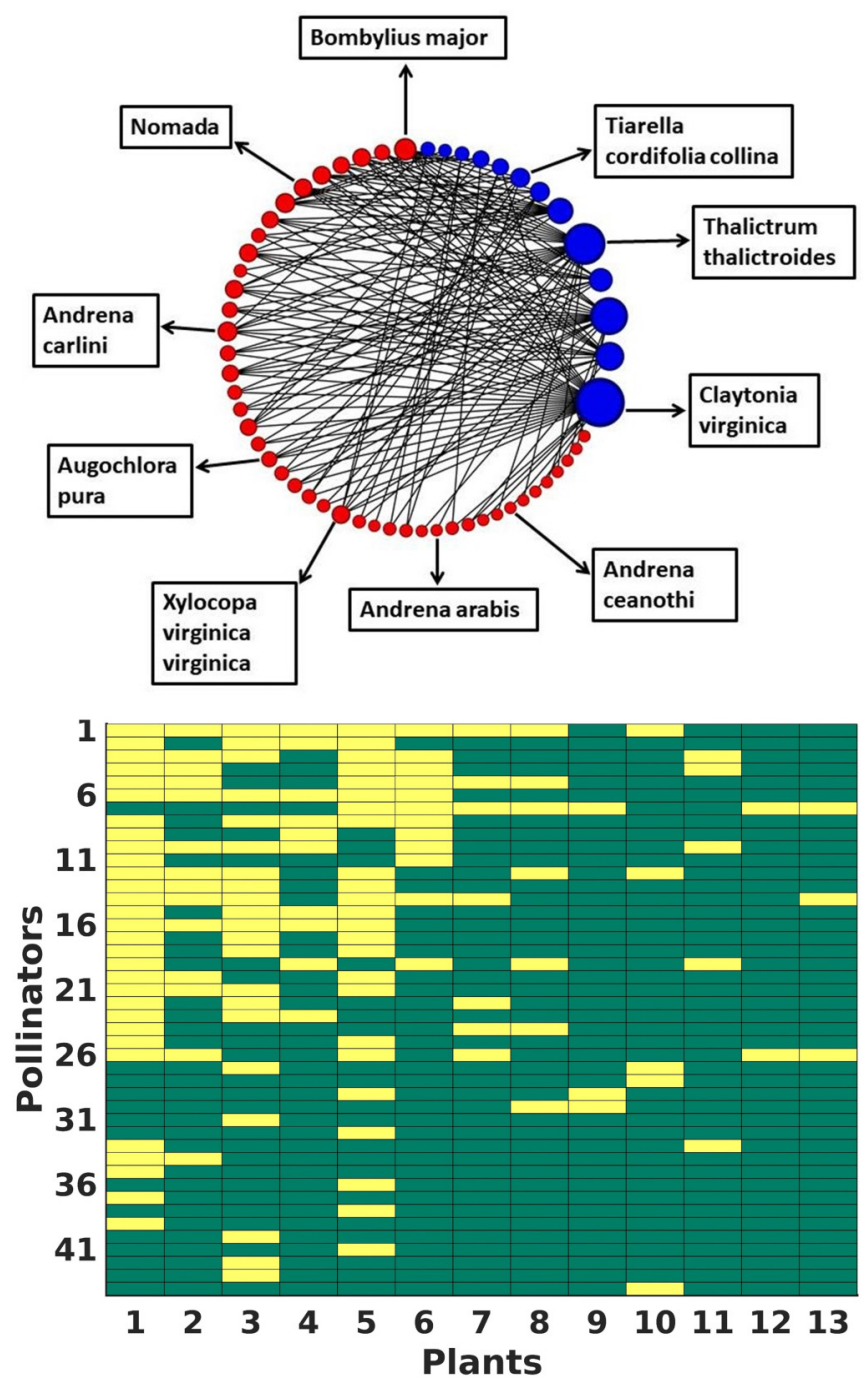

FIG. 2. Illustration of a representative real-world mutualistic network. Upper panel: bipartite network structure of network C (from North Carolina, USA, as described in the text) with $S_{X}=$ 44 pollinators (red dots), $S_{Y}=13$ plants (blue dots), and $L=143$ mutualistic links (black lines). The size of a red or a blue dot is proportional to the degree of this node. The actual photo images of seven pollinator and three plant species are presented. Lower panel: matrix representation of the network, where the vertical and horizontal indices represent pollinator and plant species, respectively. A yellow cell means the existence of a specific mutualistic link while a dark green cell indicates lack of such a link. Species are ordered according to their number of interactions.

will not go down again unless the noise amplitude becomes unreasonably large. From the point of view of nonlinear dynamics, this is a multistable system since there are two attractors for $\gamma>\gamma_{t p}$ with their own basins of attraction: one is the high-abundance stable state corresponding to the normal state, and another is the low-abundance stable state corresponding to the extinction state. The reason for species to remain in the high-abundance stable state lies in the bistable nature of the dynamics away from the tipping point. The relationship between the noise strength and mutualistic interactions allows for hopping between both stable asymptotic states. An unstable steady state sits between the two stable 

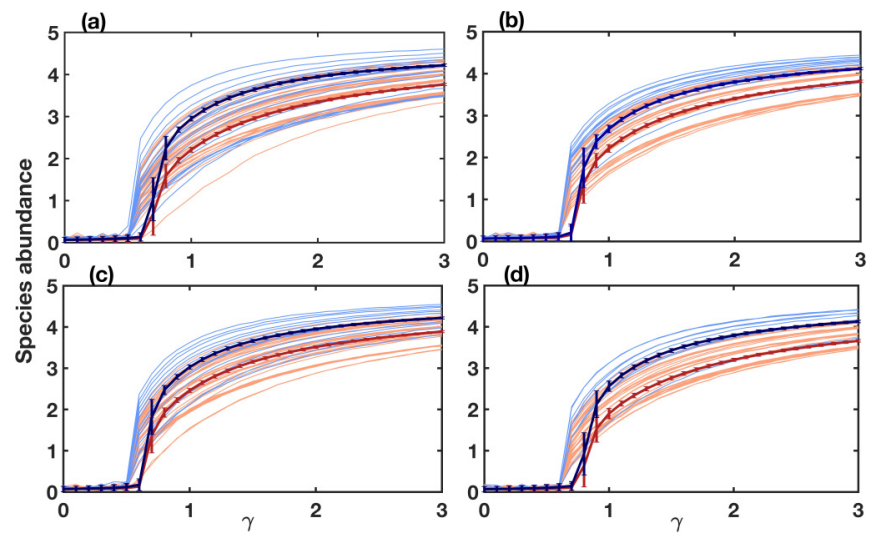

FIG. 3. Noise-enabled species recovery for the four empirical mutualistic networks. (a)-(d) The recovery curves of species abundances for networks A-D described in the text, respectively. The light red and light blue curves are the pollinator and plant abundances versus the normalized mutualistic interaction strength, respectively, as it is increased from a value below the tipping point (in the aftermath of a tipping point transition). The transition points for networks A-D are approximately $0.45,0.54,0.47$, and 0.58 , respectively. The parameter values of the full system are $\alpha_{i}^{(X)}=\alpha_{i}^{(Y)}=-0.1$, $\beta_{i i}^{(X)}=\beta_{i i}^{(Y)}=1, h=0.2, \rho=0.5, \mu_{X}=10^{-4}$, and $\mu_{Y}=10^{-4}$. The noise amplitude is $\sigma=0.1$. The values of the species abundances are collected after a long simulation time $(T=400)$ that guarantees the convergence of the networked system to a stable steady state. The dark red and blue curves with error bars are the results from the corresponding 2D reduced model. The generic parameter values of the reduced model (same for all the networks) are $\alpha=-0.1, \beta=1$, $h=0.2, \mu=0.0001$, and $\sigma=0.1$, and the initial conditions are $X(0)=10^{-3}$ and $Y(0)=10^{-3}$. The effective mutualistic parameters for the four empirical networks are (A) $\left\langle\gamma_{x}\right\rangle=12.638$ and $\left\langle\gamma_{y}\right\rangle=$ 5.118, (B) $\left\langle\gamma_{x}\right\rangle=10.036$ and $\left\langle\gamma_{y}\right\rangle=5.672$, (C) $\left\langle\gamma_{x}\right\rangle=12.118$ and $\left\langle\gamma_{y}\right\rangle=6.092$, and (D) $\left\langle\gamma_{x}\right\rangle=12.638$ and $\left\langle\gamma_{y}\right\rangle=5.118$.

steady states serving as the boundary separating the two basins of attraction. The tipping point transition marks the collision between the unstable state and the stable state species abundances. When the system approaches asymptotically a stable state, if the external interference of the system has not been increased, it will not cross the boundary to approach another state. As a result, the species are kept in the high-abundance stable state after transiting through the tipping point in the recovery process.

Examples of the algebraic scaling law (1) are shown in Fig. 5, where five sets of data points and their linear fits from both the full network and the reduced model are shown for five respective values of the intrinsic growth rate $\alpha$. The reason to choose negative $\alpha$ values is that, when the species are in an extinction state, all the abundances are zero or nearly zero. In an adversarial environment with no mutualistic interactions, a negative growth rate is more ecologically meaningful. For instance, intensive agricultural activities, changes in land use, pesticides, alien invasive species, diseases, pests, and climate change-all these impacts in a local ecological system will cause a negative growth of pollinators.

The impacts of plants and pollinators in the natural environment are different. To address this issue, we compare two different conditions: (a) plants having smaller $\sigma$ values than

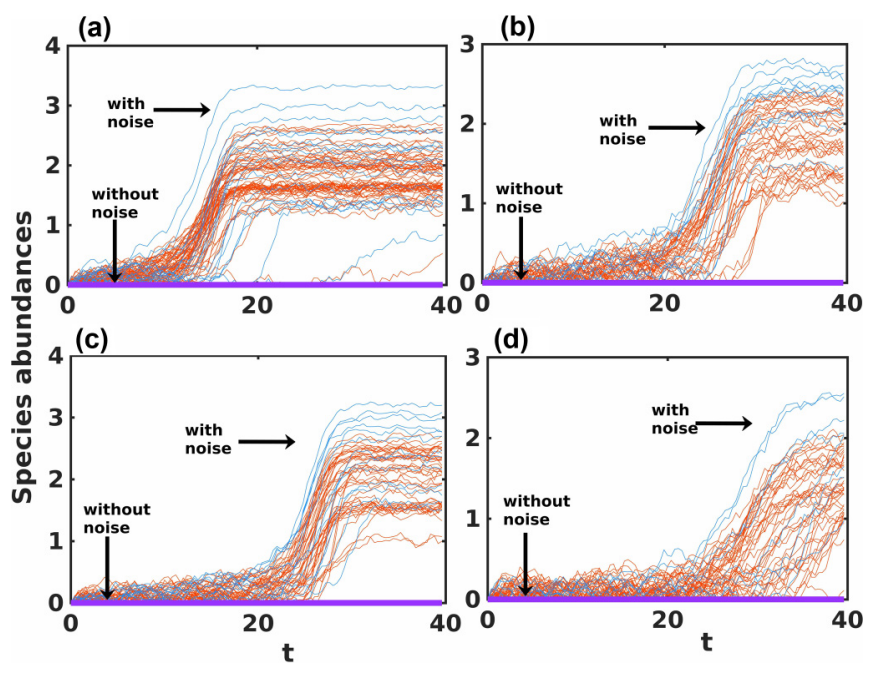

FIG. 4. Examples of noise-enabled species recovery. Panels (a)(d) correspond to networks A-D, respectively. For a given network, the time series of all pollinators (red curves) and plants (blue curves) are shown, where the initial abundances of the species are near-zero values determined by the small migration effect (corresponding to the low-abundance stable state; see Fig. 10 below), which are randomly chosen from the small interval $\left[0,10^{-3}\right]$. For all cases, the value of the normalized mutualistic interaction strength is $\gamma=0.8$. Without noise, species recovery does not occur because all the abundances remain at near zero values. We show the recovery behavior for $\sigma=$ 0.1 . The generic network parameters are $\alpha_{i}^{(X)}=\alpha_{i}^{(Y)}=-0.1, \beta_{i i}^{(X)}=$ $\beta_{i i}^{(Y)}=1, h=0.2, \rho=0.5, \mu_{X}=0.0001$, and $\mu_{Y}=0.0001$.

pollinators and (b) individual species in the empirical networks having different $\sigma$ values, e.g., chosen from the range $\left[10^{-4}, 10^{-1}\right]$. Figure 6 shows the recovery process for plants and pollinators under different noise strengths. In mutualistic networks, pollinators are insects like bees, which are more environmentally affected than most plants. We thus associate small noise strength with plants and larger noise with pollinators. For illustrative purposes, we consider three combinations of plant and pollinator noise: $\left(10^{-2}, 10^{-1}\right),\left(10^{-3}, 10^{-1}\right)$, and $\left(10^{-4}, 10^{-1}\right)$. For these three cases, while the recovery slopes are slightly different, the species abundances and the essential results remain unaffected. We also consider a more complex situation where, in the natural state, species receive different noise inputs, which is implemented by choosing the noise strength of each species in the empirical network randomly from the range $\left[10^{-4}, 10^{-1}\right]$. Calculating the recovery and collapse curves for the four empirical networks, we obtain essentially the same results, indicating that the characteristic features of species recovery and collapse are not affected by the amount of noise received, provided that the noise strength is within a reasonable range.

\section{Random mutualistic networks}

We address the issue of nestedness in ecological networks by comparing the recovery dynamics between random and empirical mutualistic networks. Nestedness is biogeographic, and it is another important characteristic of empirical networks $[38,69,70]$, which has been associated with the mutualistic interactions from different species. It was thought that nestedness can promote biodiversity in mutualistic systems 

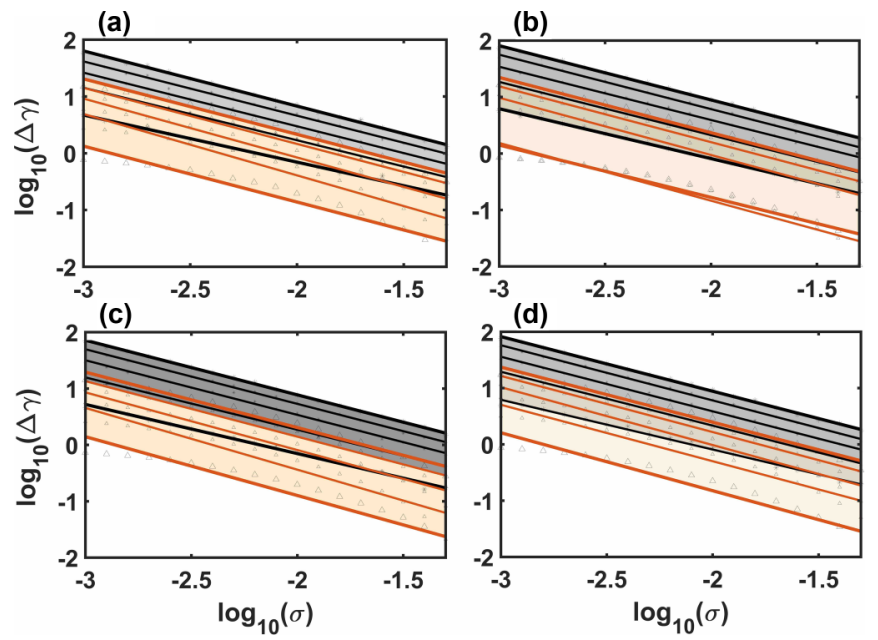

FIG. 5. Scaling law quantifying noise-enabled species recovery in complex mutualistic networks. We show the scaling law (1) for networks A-D in panels (a)-(d), respectively, where the relevant quantities $\Delta \gamma$ and $\sigma$ are displayed on a logarithmic scale. For each network, two sets of data are shown: those from the full network model (black) data points and the corresponding linear fits and those from the reduced model (orange) data points and the corresponding linear fits). For each set, five different cases corresponding to five different values of $\alpha, \alpha_{i}^{(X)}=\alpha_{i}^{(Y)}=-0.5,-0.4,-0.3,-0.2,-0.1$ for the full network model and $\alpha=-0.5,-0.4,-0.3,-0.2,-0.1$ for the reduced model, are shown. The generic network parameters are $\beta=1, \beta_{i i}^{(X)}=\beta_{i i}^{(Y)}=1.0, h=0.2$, and $\rho=0.5$. For each value of the noise amplitude $\sigma$, simulations are carried out for a long time interval $(T=400)$, which guarantees that the system has approached a stable steady state by then. The reasonably good linear fits suggest the algebraic scaling law (1), and the partial overlap between the black and orange regions indicates the ability of the reduced model to capture the essential dynamics of the full networked system.

through increasing the capability of the pollinator populations to withstand harsh conditions. In Ref. [38] a spectral graph approach was proposed based on the quality of bipartite networks to study nestedness, with the result that nested mutualistic networks have the minimal stability. Another result was that the binary nested quantitative preference of complex ecological networks is non-nested, indicating limited pollinator (a)

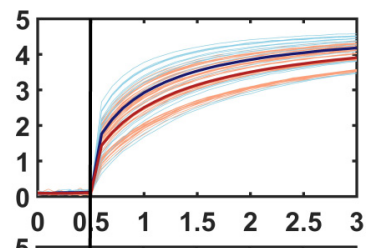

(b) 5
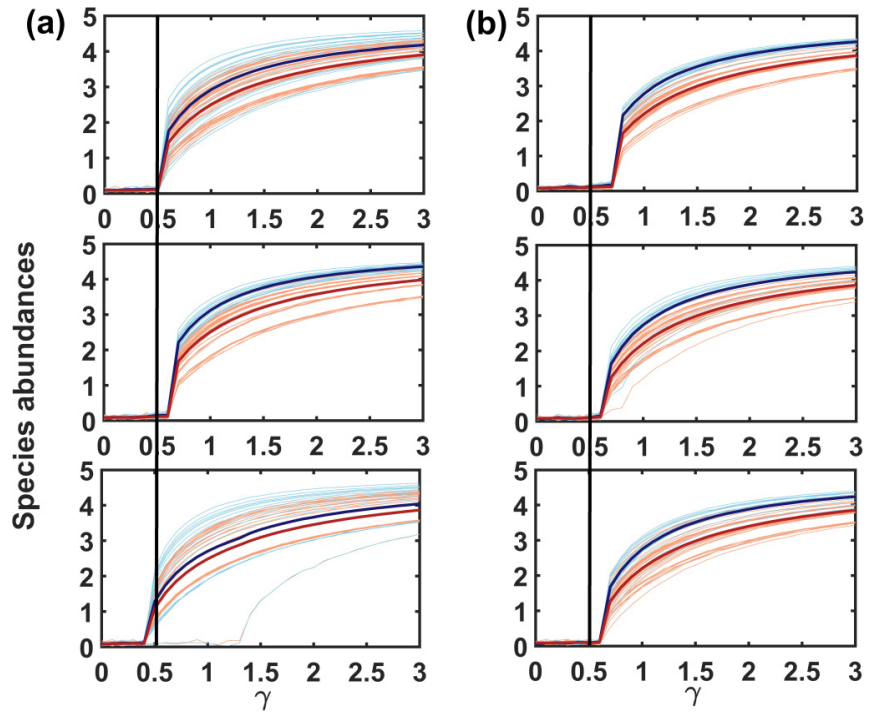

FIG. 7. Noise-induced recovery in random networks with different numbers of plant and pollinator species. $(a, b)$ Recovery curves of species abundances for two different random networks: one with 61 pollinators and 17 plants, and another with 44 pollinator and 13 plants. The light red and light blue curves are the pollinator and plant abundances versus the normalized mutualistic interaction strength, respectively. The parameter values are $\alpha_{i}^{(X)}=\alpha_{i}^{(Y)}=-0.1$, $\beta_{i i}^{(X)}=\beta_{i i}^{(Y)}=1.0, h=0.2, \rho=0.5$, and $\mu_{X}=\mu_{Y}=10^{-4}$. The noise strength is $\sigma=0.1$. The values of nestedness from the top to the bottom panel are $0.2,0.5$, and 0.7 respectively. The dark red and blue curves are the average abundance of pollinators and plants, respectively.

overlap of favored plants in mutualistic networks. Ecological systems could avoid species competition by dividing the species preferences with the condition of binary constraint, which is beneficial to resource allocation in the system.

We study the difference among random networks with different numbers of species, as shown in Fig. 7. As the level of nestedness [38] is increased (see Appendix B for a method to systematically vary the network nestedness), the value of $\gamma$ in the recovery process decreases. For networks with different species abundances, the recovery process is similar: in the presence of noise, a tipping point transition occurs when the
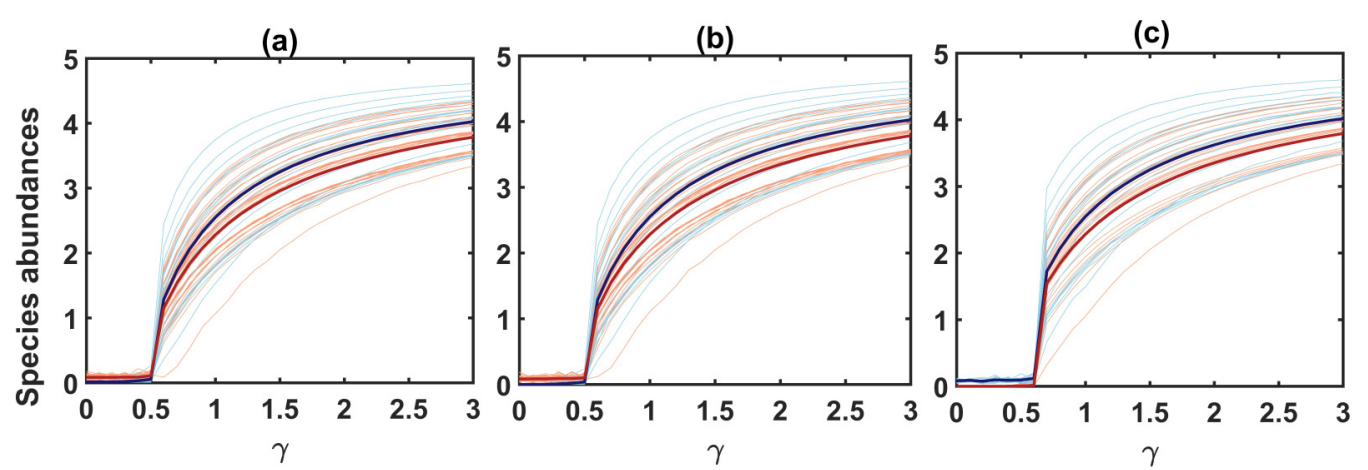

FIG. 6. Recovery process when pollinator and plant species are subject to different levels of noise. For empirical network A, pollinator (light red) and plant (light blue) abundances versus the normalized mutualistic interaction strength under the following three combinations of plant and pollinator noise: (a) $\left(10^{-2}, 10^{-1}\right)$, (b) $\left(10^{-3}, 10^{-1}\right)$, and (c) $\left(10^{-4}, 10^{-1}\right)$. Parameter values are $\alpha_{i}^{(X)}=\alpha_{i}^{(Y)}=-0.1, \beta_{i i}^{(X)}=\beta_{i i}^{(Y)}=$ 1.0, $h=0.2, \rho=0.5$, and $\mu_{X}=\mu_{Y}=10^{-4}$. The dark red and blue curves are the average abundance of plants and pollinators, respectively. 


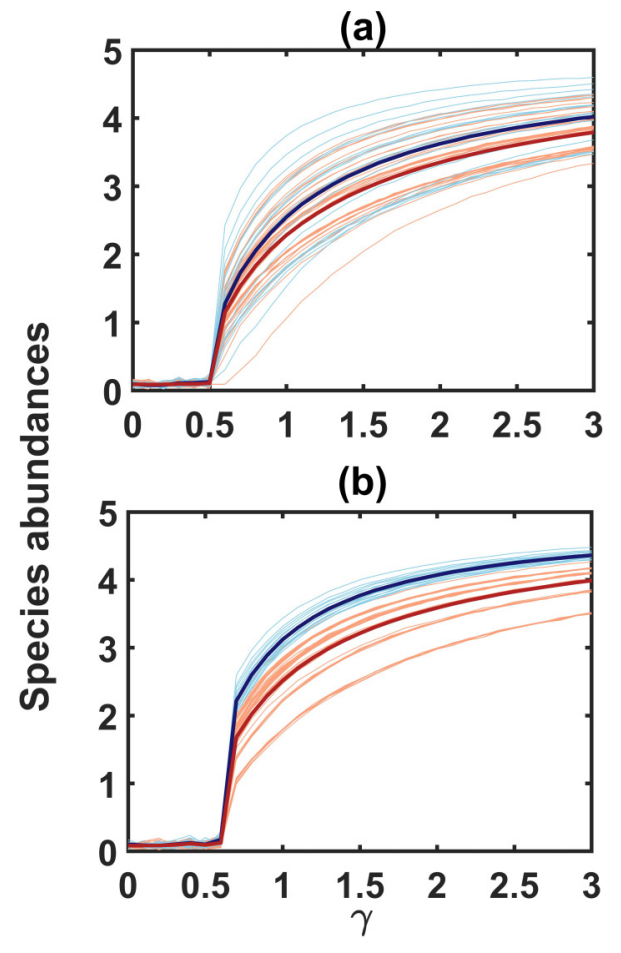

FIG. 8. Comparison between recovery process in random and empirical network. (a, b) Recovery curves of an empirical network and a random network with the same numbers of species, respectively. The light red and light blue curves are the pollinator and plant abundances versus the normalized mutualistic interaction strength, respectively. The parameter values are $\alpha_{i}^{(X)}=\alpha_{i}^{(Y)}=-0.1$, $\beta_{i i}^{(X)}=\beta_{i i}^{(Y)}=1.0, h=0.2, \rho=0.5$, and $\mu_{X}=\mu_{Y}=10^{-4}$. The noise strength is $\sigma=0.1$. The dark red and blue curves are the average abundance of plants and pollinators.

value of $\gamma$ decreases through a critical value $\gamma_{c}$ that depends on the noise amplitude $\sigma$. For a random network with two groups of species, the dynamical behavior is qualitatively the same as that of the empirical network. However, as the network becomes more nested, the critical parameter value causing the network collapse becomes smaller, and the value of the network recovery point also becomes smaller.

A comparison between random and empirical networks is illustrated in Fig. 8. The system recovery does not change as one considers different networks, and the scaling law between $\Delta \gamma$ and the noise strength $\sigma$ does not change, as shown in Fig. 9, where the red line is for the empirical network, and the light blue and dark blue lines are for random networks with strong and weak nestedness, respectively. The scaling between $\Delta \gamma$ and $\sigma$ for the random networks is algebraic, as in the case of empirical networks.

For different empirical networks, the conditions for network recovery are different. However, the number of links is not the reason for the differences. For instance, for $\alpha_{i}^{(X)}=$ $\alpha_{i}^{(Y)}=-0.1$ and $\sigma=0.1$, the global recovery points for four empirical networks are $0.378,0.394,0.331,0.354$ for networks A-D, respectively, where the respective numbers of mutualistic links are 146, 106, 143, and 42. There is no direct connection between the number of mutualistic links and the recovery process.

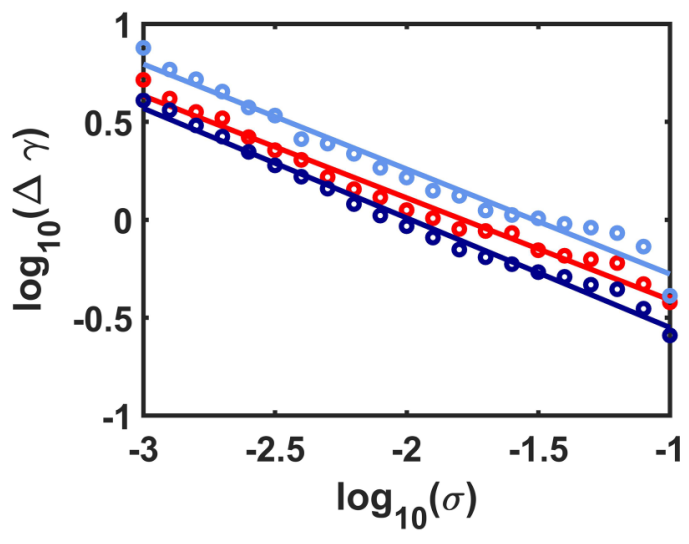

FIG. 9. Comparison between scaling laws with noise-enabled species recovery in empirical and random mutualistic networks. The red line is for an empirical network, and the light blue and dark blue lines are for random networks with a large and a small value of nestedness, respectively. The parameter values are $\alpha_{i}^{(X)}=\alpha_{i}^{(Y)}=$ $-0.1, \beta_{i i}^{(X)}=\beta_{i i}^{(Y)}=1.0, h=0.2, \rho=0.5$, and $\mu_{X}=\mu_{Y}=10^{-4}$. The noise strength is $\sigma=0.1$.

\section{B. Theoretical understanding of the scaling law}

The key to developing a theoretical understanding of the noise scaling law (1) is the fact that the generic dynamical mechanism for the emergence of a tipping point, regardless of the system dimension, is a saddle-node bifurcation, as shown schematically in Fig. 10 where, for illustrative purposes, the bifurcation parameter is taken to be the normalized

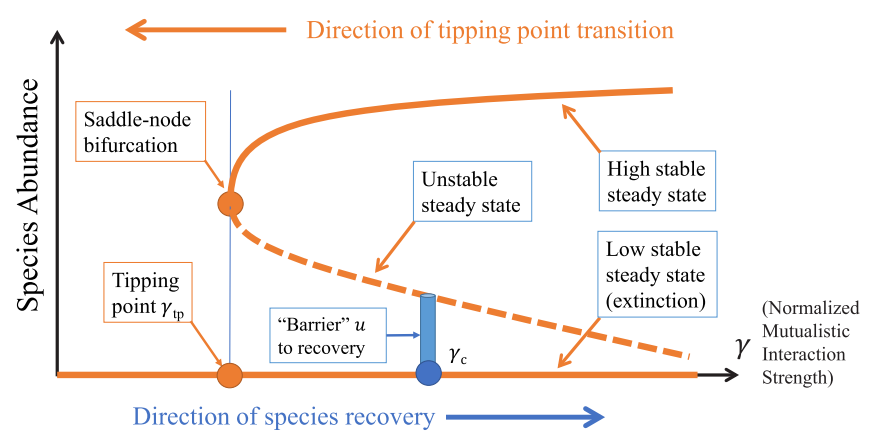

FIG. 10. A schematic illustration of the nonlinear dynamical origin of a tipping point transition and the mechanism of noiseenabled species recovery. The generic dynamical origin of a tipping point transition in a complex mutualistic network, in spite of its high dimension, is a saddle-node bifurcation at the transition point that creates a high-abundance state (a high-abundance stable state) and an unstable steady state for $\gamma>\gamma_{t p}$. The extinction state (a low-abundance stable state) is a stable fixed point of the system that exists in the whole range of the value of the mutualistic interaction strength. For $\gamma>\gamma_{t p}$, there is bistability in the system in that there are two coexisting stable steady states: one high and another low, which are "separated" by the unstable steady state. (More precisely, in the full phase space, the stable manifold of the unstable steady state is the boundary that separates the basins of attraction of the lowand high-abundance stable state.) As illustrated, in order to enable species recovery, fluctuations in its abundance must be larger than the value of the unstable steady state ("overcoming" the barrier), which can be achieved with noise. 
mutualistic interaction strength $\gamma$. The bifurcation occurs in the direction of increasing the value of $\gamma$, where the bifurcation point defines the tipping point $\gamma_{t p}$. For $\gamma<\gamma_{t p}$, the system possesses only one stable steady state, one corresponding to the extinction state in which the species abundances assume near-zero values that are determined by the small migration effect. For $\gamma>\gamma_{t p}$, there are three steady states in the system: two stable and one unstable, where the lowabundance stable state is a continuation of the extinction state from $\gamma<\gamma_{t p}$, and the high-abundance stable state and the unstable states are created at the saddle-node bifurcation. There is then multistability [71-79] in the system in that there are two attractors in the system for $\gamma>\gamma_{t p}$, each with its own basin of attraction. In general, the boundary separating the two basins of attraction is the stable manifold of the unstable steady state $[80,81]$.

The dynamical origin of a tipping point transition can then be understood, as follows. Say the environmental condition is such that $\gamma$ is above the critical value $\gamma_{t p}$ and the system is in the high-abundance stable state. Deterioration of the environment weakens the mutualistic interaction, causing a gradual decrease in the parameter $\gamma$. Insofar as the value of $\gamma$ stays above $\gamma_{t p}$, the system remains in the high-abundance stable state in spite of a small decrease in the overall abundance value. When $\gamma$ decreases through the critical point $\gamma_{t p}$, the high-abundance stable state disappears altogether with its basin of attraction, leaving the low-abundance stable state as the only attractor in the system with almost the entire available phase space as its basin of attraction. The system evolves into the extinction state with the abundances of all its species decreasing to near-zero values in a relatively short time.

Now consider the recovery process. In the aftermath of the tipping point transition, the system is already in the lowabundance stable state. As the value of $\gamma$ is increased through $\gamma_{t p}$, without any external influence, the system will remain in this extinction state in spite of the coexistence of the highabundance stable state created at the saddle-node bifurcation. In order for the species abundances to recover, some external perturbation, e.g., noise, is needed to drive the system out of the extinction state into the basin of the high-abundance stable state. To accomplish this, the noise amplitude must be sufficiently large to overcome the "barrier," effectively the phase space "distance" between the extinction state and the unstable steady state, as shown in Fig. 10. Since the height of this barrier decreases with $\gamma$, for a larger value of $\gamma$ the required noise amplitude for recovery is smaller. Likewise, noise of a larger amplitude will enable recovery at a point of $\gamma$ (denoted as $\gamma_{c}$ in Fig. 10) closer to the tipping point. Thus, qualitatively, we expect $\Delta \gamma=\gamma_{c}-\gamma_{t p}$ to decrease as the noise amplitude is increased.

Based on the nonlinear dynamical processes depicted in Fig. 10, we can derive the scaling law (1), as follows. Let $u$ be the height of the barrier that the system must overcome with the aid of noise as seen in Fig. 6. Under the influence of Gaussian white noise, in principle this will happen for arbitrarily small noise amplitude if an infinite amount of time is allowed. For the system to recover in a finite time, the probability that the noise perturbation exceeds $u$ must be appreciable. For white noise, this probability is given by

$$
\varepsilon=\frac{1}{\sqrt{2 \pi \sigma}} \int_{u}^{\infty} \exp \left(-\frac{x^{2}}{2 \sigma^{2}}\right) d x=\frac{1}{2} \operatorname{erfc}(c u),
$$

where $\operatorname{erfc}(\cdot)$ is the complementary error function and

$$
c \equiv 1 /(\sqrt{2} \sigma) \text {. }
$$

Using the following representation of the complementary error function [82]:

$$
\operatorname{erfc}(x) \geqslant \frac{1}{2} \sqrt{\frac{2 e}{\pi}} \frac{\sqrt{\chi-1}}{\chi} e^{-\chi x^{2}},
$$

where $\chi>1$ is a constant, we obtain

$$
\frac{1}{2} \sqrt{\frac{2 e}{\pi}} \frac{\sqrt{\chi-1}}{\chi} \exp \left[-\chi(c u)^{2}\right] \lesssim \varepsilon .
$$

This gives

$$
(c u)^{2} \gtrsim|\ln \delta|
$$

where

$$
\delta \equiv \sqrt{\frac{2 \pi}{e}} \frac{\chi}{\sqrt{\chi-1}} \varepsilon .
$$

Utilizing the definition of the constant $c$ in Eq. (11), we get

$$
\frac{u}{\sigma} \gtrsim \sqrt{2|\ln \delta|}
$$

Since $\varepsilon \gtrsim 0$, we have $\delta \gtrsim 0$. The right side of Eq. (13) is thus a constant on the order of unity.

As postulated in Fig. 10, the barrier height $u$ depends on $\Delta \gamma$. What is the scaling relation between the two quantities? To address this question, we have carried out a stability analysis of the reduced model (see Appendix A), which gives implicit but algebraically sophisticated relations between the pollinator $\left(U_{x}\right)$ and plant $\left(U_{y}\right)$ abundances and $\Delta \gamma$. Numerical solutions of these relations for five representative values of $\alpha$ (the same set of $\alpha$ values as in Fig. 5) are shown in Figs. 11 and 12 for the dependence of $U_{x}$ and $U_{y}$ on $\Delta \gamma$, respectively, from the corresponding reduced models of the four real-world networks. In all cases, the dependence can be described by an algebraic scaling law:

$$
U_{x} \text { or } U_{y} \sim(\Delta \gamma)^{-q},
$$

for $\Delta \gamma$ not close to zero, where $q>0$ is the algebraic scaling exponent. Substituting Eq. (14) into Eq. (13), we obtain the algebraic scaling law (1), with the scaling exponent $p=1 / q$. Extensive numerical evidence supporting this inverse relationship between the algebraic exponents in the scaling laws (1) and (14) is presented in Figs. 13 and 14, where the exponent $q$ is obtained from the unstable steady solution of the pollinator and plant species of the reduced model, respectively.

\section{DEMOGRAPHIC NOISE}

Demographic stochasticity is of particular importance to ecological systems [83-85] due to the random nature of the biological processes such as birth, death, and mutual interactions. Here we study the effect of demographic noise on species recovery in mutualistic networks. 

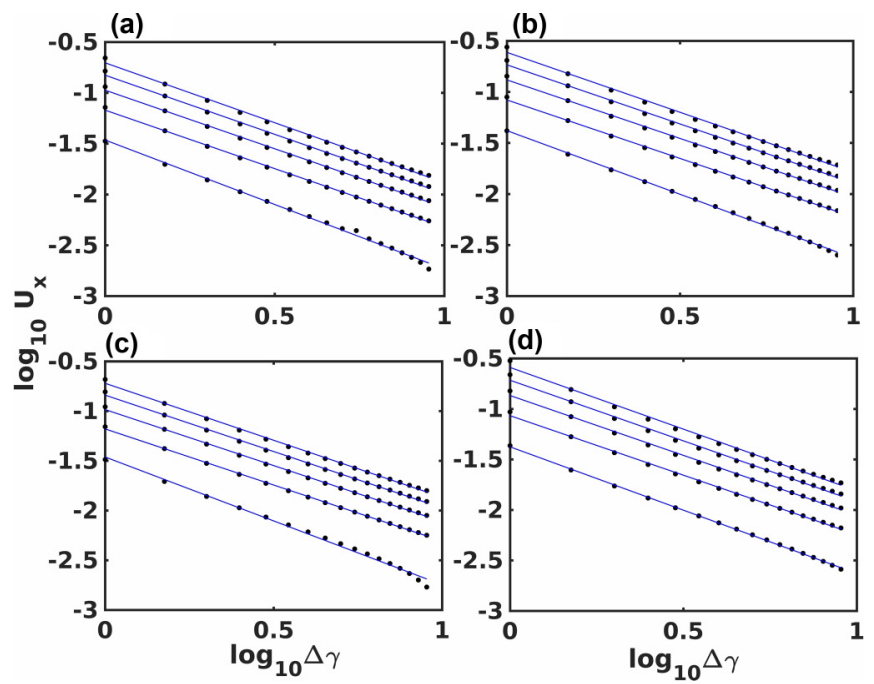

FIG. 11. Scaling of the pollinator abundance associated with the unstable steady state with the strength of mutualistic interaction beyond the tipping point. Panels (a)-(d) correspond to networks A-D, respectively. In each panel, the dependence of $U_{x}$ on $\Delta \gamma$ for five values of $\alpha$ is displayed. Different lines represent different values of $\alpha$. In all cases, the dependence can be fitted by an algebraic scaling law with the exponent $q$, which assumes values close to unity.

A demographic stochastic process is a type of multiplicative noise with amplitude proportional to the square root of the fluctuating field due to uncertainties such as the timing of birth and death and the interaction relations among the species. Demographic stochastic effects depend on the intrinsic uncertainties associated with individuals' reproduction, survival, and dispersal and are most influential in small populations. Mathematically, demographic noise depends on the dynamical variables of the system, i.e., the abundances in our mutualistic networked system. Under demographic noise, the governing equations of a mutualistic network can be
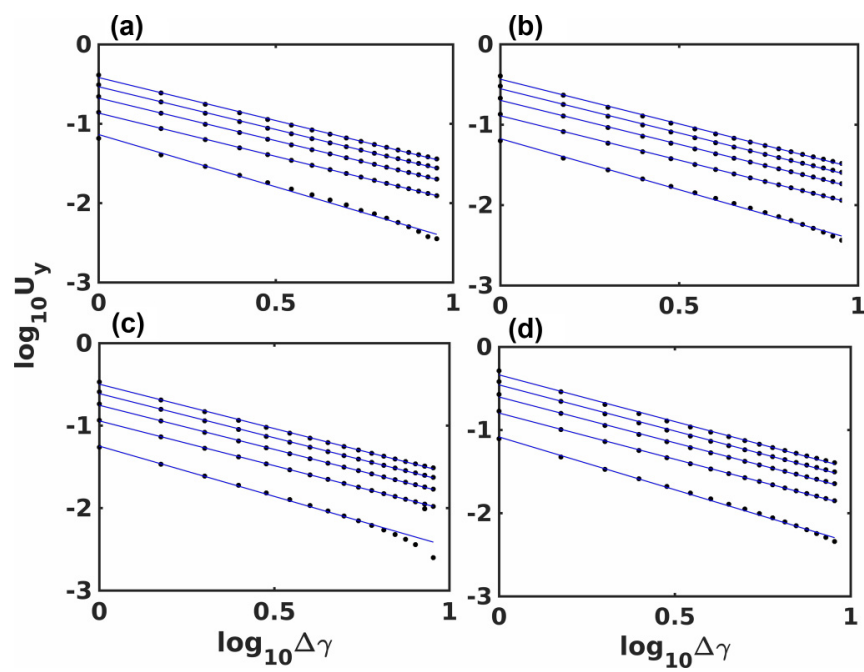

FIG. 12. Scaling of the plant abundance associated with the unstable steady state with the strength of mutualistic interaction above the tipping point value. Legends are the same as in Fig. 11.
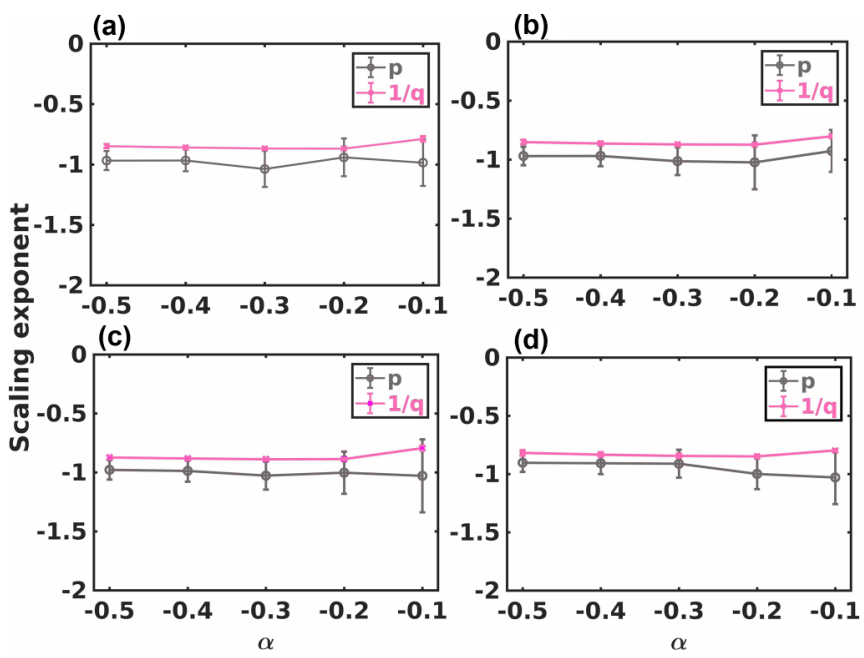

FIG. 13. Comparison between the algebraic exponent $p$ from the scaling law (1) and the inverse of the algebraic exponent $q$ from (14) for the effective pollinator abundance. (a-d) Five values of the exponent $p$ for five different values of $\alpha$ in comparison with the corresponding values of $1 / q$ from the effective unstable pollinator abundance in the reduced model for networks A-D, respectively.

written as

$$
\begin{aligned}
\frac{d X_{i}}{d t}= & \alpha_{i}^{(X)} X_{i}-\sum_{j=1}^{S_{X}} \beta_{i j}^{(X)} X_{i} X_{j}+\mu_{X} \\
& +\frac{\sum_{k=1}^{S_{Y}} \gamma_{i k}^{(X)} Y_{k}}{1+h \sum_{k=1}^{S_{Y}} \gamma_{i k}^{(X)} Y_{k}} X_{i}+R^{-1} \sqrt{X_{i}} \zeta_{i}(t), \\
\frac{d Y_{i}}{d t}= & \alpha_{i}^{(Y)} Y_{i}-\sum_{j=1}^{S_{Y}} \beta_{i j}^{(Y)} Y_{i} Y_{j}+\mu_{Y} \\
& +\frac{\sum_{k=1}^{S_{X}} \gamma_{i k}^{(Y)} X_{k}}{1+h \sum_{k=1}^{S_{X}} \gamma_{i k}^{(Y)} X_{k}} Y_{i}+R^{-1} \sqrt{Y_{i}} \nu_{i}(t)
\end{aligned}
$$
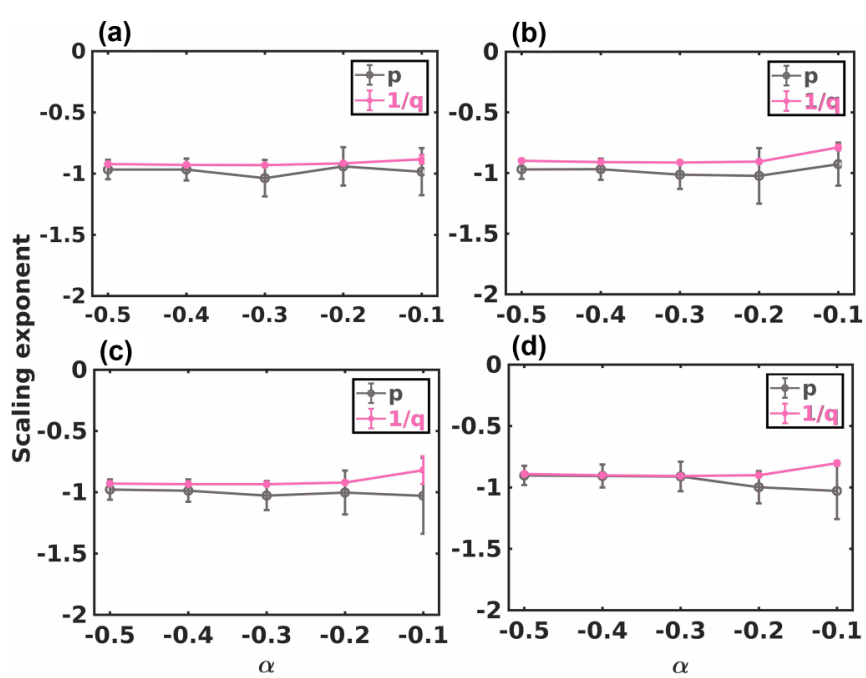

FIG. 14. Comparison between the algebraic exponent $p$ from the scaling law (1) and the inverse of the algebraic exponent $q$ from (14) for the effective plant abundance. Same legends as in Fig. 13 except that the inverse exponent $1 / q$ is calculated based on the effective unstable plant abundance. 

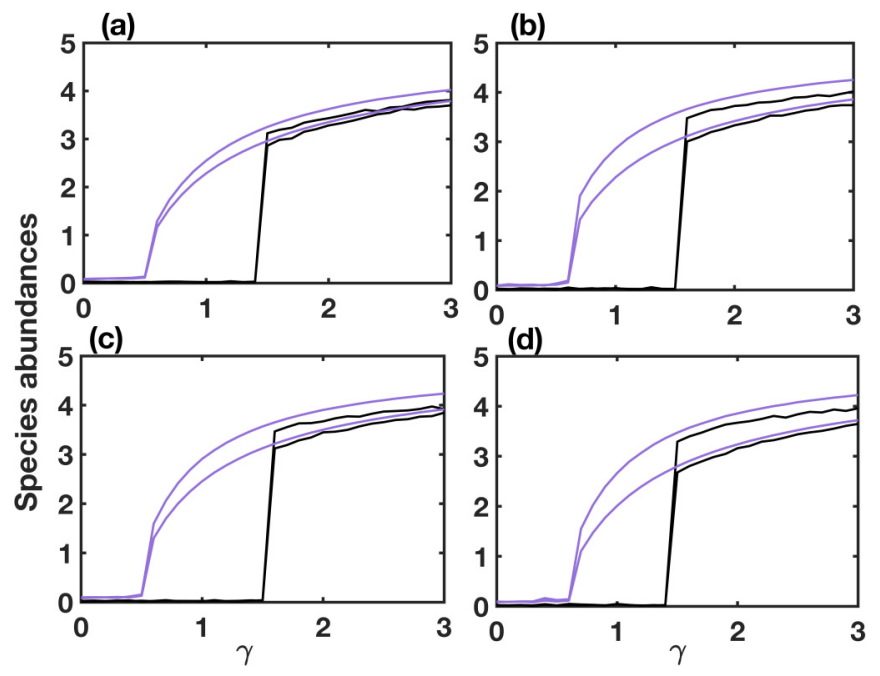

FIG. 15. Noise-enabled species recovery for four empirical mutualistic networks with additive white noise and demographic noise. The purple and black curves are average abundances for all species with additive white noise (of amplitude 0.1 ) and demographic noise, respectively, versus the normalized mutualistic interaction strength as it is increased from a value below the tipping point (in the aftermath of a tipping point transition). The transition points for networks A-D under additive white noise are approximately $0.45,0.54$, 0.47 , and 0.58 , respectively. The transition points for networks A-D with demographic noise are approximately $1.4,1.5,1.5$, and 1.4, respectively. The values of the transition point under demographic noise are significantly larger than those under white noise. The system parameter values are $\alpha_{i}^{(X)}=\alpha_{i}^{(Y)}=-0.1, \beta_{i i}^{(X)}=\beta_{i i}^{(Y)}=1$, $h=0.2, \rho=0.5, \mu_{X}=10^{-4}, \mu_{Y}=10^{-4}, \sigma=0.1$, and $R=4$. The values of the species abundances are collected after a relatively long transient time $(T=400)$ at which the system has well converged to a stable steady state. The initial conditions are $X(0)=10^{-3}$ and $Y(0)=10^{-3}$. All the simulations are based on 100 statistical realizations.

where $R$ is the order of magnitude of the population size or the area of habitat in demographic randomness, which affects the abundances of individual species, and $\zeta_{i}(t)$ and $v_{i}(t)$ are white noise processes of zero mean and unit variance. The multiplicative terms $R^{-1} \sqrt{X_{i}} \zeta_{i}(t)$ and $R^{-1} \sqrt{Y_{i}} v_{i}(t)$ represent demographic noises with state-dependent correlations. We numerically solve Eqs. (15) and (16) using the standard second-order method for integrating stochastic differential equations $[85,86]$.

The deterministic system underlying Eqs. (15) and (16) possesses two stable steady states: one being the lowabundance or extinction state that occurs for $\gamma<\gamma_{t p}$ and another being the high-abundance state for $\gamma>\gamma_{t p}$ (denoted as $X_{h}$ and $Y_{h}$ ). Under demographic noise, the abundances of the pollinator and plant species fluctuate about one of the steady states value with the standard deviations $\sqrt{X_{h}}$ and $\sqrt{X_{h}}$, respectively. Figure 15 shows a representative comparison between the effects of additive white noise and demographic noise, where both types of noise can drive the system to the high-abundance state after collapse. However, the critical point of recovery with demographic noise is larger than that with additive white noise. In the initial extinction state with abundances $X(0)=10^{-3}$ and $Y(0)=10^{-3}$, the demographic noises are weak. After recovery, there are large fluctuations in the species abundances, making demographic noise stronger. Comparing with the case of white noise, under demographic noise it is significantly more difficult to drive the system out of the extinction state into the basin of the high-abundance state. The main reason is that, when the system is in the extinction state, the abundances are close to zero, rendering infinitesimally small the amplitude of demographic noise. As a result, practically the noise has little effect on the dynamics and thus is not beneficial to species recovery. In order to recover, much stronger mutualistic interaction is necessary. Because of the dependence of the amplitude of demographic noise on the state variables, a scaling law such as (1) for the case of white noise cannot be defined.

\section{DISCUSSION}

The benefits of noise to nonlinear dynamical systems from the viewpoints of understanding certain natural phenomena and of engineering applications such as signal processing have been known and extensively studied since the discovery of the phenomenon of stochastic resonance [17-23] where, counterintuitively, a certain amount of deliberately applied noise can enhance and maximize the signal-to-noise ratio of the output of the system. A related phenomenon is noise-induced frequency [24] or coherence resonance [25-28] where noise can be exploited to improve, sometimes significantly, the temporal regularity of the output signal of a nonlinear oscillator by inducing or enhancing a dominant frequency component in its Fourier power spectrum. Ecological systems are fundamentally nonlinear [1-3], but the approach of purely deterministic modeling may not be sufficient to describe, characterize, and understand ecological phenomena in the real world due to the ubiquitous occurrence of various random forces in nature $[4,6,10,11]$. It is thus imperative to study the interplay between deterministic nonlinearity and stochasticity in ecological systems $[5,9,12,13]$. In this regard, previous studies revealed the beneficial role of noise in promoting coexistence $[7,8]$ in a low-dimensional model of patch population dynamics of dispersing species [14-16].

The main contributions of the present work are two: (1) demonstration of the benefits of noise in high-dimensional, real-world ecological systems modeled as complex mutualistic networks, and (2) discovery of a scaling law characterizing the advantages of noise in enabling species recovery in a quantitative manner. The fundamental dynamics of an ecological network with mutualistic interactions between two groups of species, e.g., pollinators and plants, are of the Holling type [29-36,56,57]. We have focused on the situation where there is a tipping point in the networked system. In terms of generic nonlinear dynamics, a tipping point transition is essentially an inverse saddle-node bifurcation in the parameter regime where the system exhibits multistability (bistability) in spite of its intrinsic high dimensionality. In particular, in a healthy state where all species coexist, the system "lives" in a high-abundance stable state, even there is a coexisting, low-abundance stable state corresponding to extinction. The high-abundance stable state, together with an unstable steady state that provides the boundary safely separating the basin 
of the high-abundance stable state from that of the lowabundance stable state is created by a saddle-node bifurcation. As the environment deteriorates continuously, the relevant parameter changes in the opposite direction and the system moves towards the original bifurcation point at which both the high-abundance stable state and the unstable state are destroyed simultaneously, leaving the extinction state as the only stable state in the system. This signifies a tipping point transition. In the aftermath of the transition, the abundances of all the species in the system are near zero. The situation where the system is locked in the extinction state will not change without external influence or perturbations even when the environment is being improved so that the bifurcation parameter now passes through the tipping point and multistability is restored. Noise provides the necessary external influences that can drive the system out of the extinction state towards the high-abundance stable state with a nonzero probability, enabling species recovery. The larger the noise amplitude, the less demanding the extent of environmental improvement is, thereby facilitating recovery even when the system has returned to the bistability regime not far from the tipping point. Quantitatively, the ease at which noise helps species to recover is described by the scaling law (1) uncovered in this work.

The results of this work have the following implications. While the principle of noise-enabled species recovery is general, in practice it may not be feasible to "wait" for a random burst in the species abundances to occur to push the system above the critical level as determined by the unstable steady state. This is the case where, in the aftermath of a tipping point transition, the improvement in the environment is not significant enough so that the system is in the bistability regime not too much beyond the tipping point where the probability for a large burst of abundances is exponentially small so that the waiting time can be exponentially long. However, one can deliberately apply a "controlled burst" by introducing additional abundances through a balanced combination of certain species in the system. How to calculate an optimal perturbation to carry out the control strategy is an open question at the present, but the insights gained from this work, especially the dynamical analysis through the reduced model of mutualistic networks, indicate that either pollinators or plants can be controlled. However, biologically, pollinators are more accessible to control.

\section{ACKNOWLEDGMENT}

We would like to acknowledge support from the Vannevar Bush Faculty Fellowship program sponsored by the Basic Research Office of the Assistant Secretary of Defense for Research and Engineering and funded by the Office of Naval Research through Grant No. N00014-16-1-2828.

\section{APPENDIX A: STEADY-STATE SOLUTIONS AND STABILITY ANALYSIS BASED ON THE REDUCED MODEL WITHOUT MIGRATION}

The steady-state solutions of the reduced model [Eqs. (8) and (9)] are determined by

$$
\frac{d x}{d t}=\alpha x-\beta x^{2}+\frac{\left\langle\gamma_{x}\right\rangle y}{1+h\left\langle\gamma_{x}\right\rangle y} x=0
$$

$$
\frac{d y}{d t}=\alpha y-\beta y^{2}+\frac{\left\langle\gamma_{y}\right\rangle x}{1+h\left\langle\gamma_{y}\right\rangle x} y=0 .
$$

There are five steady-state solutions. The first solution is given by

$$
x_{s 1}=0, \quad y_{s 1}=\frac{\alpha}{\beta} .
$$

The second steady-state solution is

$$
\begin{aligned}
x_{s 2}= & \frac{-s_{1}+s_{2}-\left[\left(s_{1}-s_{2}\right)^{2}-4 s_{3} s_{4}\right]^{1 / 2}}{2 s_{4}}, \\
y_{s 2}= & \frac{1}{s_{5}}\left\{\left(\left\langle\gamma_{x}\right\rangle-\left\langle\gamma_{2}\right\rangle \alpha\right)-\frac{s_{6}}{2 s_{4}}\right. \\
& -\frac{\left\langle\gamma_{x}\right\rangle\left\langle\gamma_{y}\right\rangle\left[\left(s_{1}-s_{2}\right)^{2}-2 s_{3} s_{4}\right]^{1 / 2}}{2 s_{4}} \\
& -\frac{\left\langle\gamma_{x}\right\rangle\left\langle\gamma_{y}\right\rangle \alpha h\left[\left(s_{1}-s_{2}\right)^{2}-2 s_{3} s_{4}\right]^{1 / 2}}{2 s_{4}} \\
& \left.-\frac{\left\langle\gamma_{y}\right\rangle \beta\left[\left(s_{1}-s_{2}\right)^{2}-2 s_{3} s_{4}\right]^{1 / 2}}{2 s_{4}}\right\} .
\end{aligned}
$$

The third steady-state solution is

$$
\begin{aligned}
x_{s 3}= & \frac{-s_{1}+s_{2}+\left[\left(s_{1}-s_{2}\right)^{2}-4 s_{3} s_{4}\right]^{1 / 2}}{2 s_{4}}, \\
y_{s 3}= & \frac{1}{s_{5}}\left\{\left(\left\langle\gamma_{x}\right\rangle-\left\langle\gamma_{2}\right\rangle \alpha\right)-\frac{s_{6}}{2 s_{4}}\right. \\
& +\frac{\left\langle\gamma_{x}\right\rangle\left\langle\gamma_{y}\right\rangle\left[\left(s_{1}-s_{2}\right)^{2}-2 s_{3} s_{4}\right]^{1 / 2}}{2 s_{4}} \\
& +\frac{\left\langle\gamma_{x}\right\rangle\left\langle\gamma_{y}\right\rangle \alpha h\left[\left(s_{1}-s_{2}\right)^{2}-2 s_{3} s_{4}\right]^{1 / 2}}{2 s_{4}} \\
& \left.+\frac{\left\langle\gamma_{y}\right\rangle \beta\left[\left(s_{1}-s_{2}\right)^{2}-2 s_{3} s_{4}\right]^{1 / 2}}{2 s_{4}}\right\},
\end{aligned}
$$

where

$$
\begin{aligned}
s_{1}= & \left\langle\gamma_{x}\right\rangle\left\langle\gamma_{y}\right\rangle+\left\langle\gamma_{x}\right\rangle\left\langle\gamma_{y}\right\rangle \alpha h-\left\langle\gamma_{x}\right\rangle\left\langle\gamma_{y}\right\rangle \alpha h^{2}, \\
s_{2}= & \left\langle\gamma_{x}\right\rangle \alpha \beta h-\left\langle\gamma_{y}\right\rangle \alpha \beta h+\beta^{2}, \\
s_{3}= & \left\langle\gamma_{x}\right\rangle \alpha+\left\langle\gamma_{x}\right\rangle \alpha^{2} h+\alpha \beta, \\
s_{4}= & -\left\langle\gamma_{x}\right\rangle\left\langle\gamma_{y}\right\rangle \beta-\left\langle\gamma_{x}\right\rangle\left\langle\gamma_{y}\right\rangle h^{2} \alpha \beta-\left\langle\gamma_{y}\right\rangle h \beta^{2}, \\
s_{5}= & \left\langle\gamma_{x}\right\rangle\left\langle\gamma_{y}\right\rangle+\left\langle\gamma_{x}\right\rangle\left\langle\gamma_{y}\right\rangle h \alpha+\left\langle\gamma_{x}\right\rangle \beta, \\
s_{6}= & \left\langle\gamma_{x}\right\rangle^{2}\left\langle\gamma_{y}\right\rangle^{2}\left(1-3 h \alpha+3 h^{3} \alpha^{2}+s_{7}\right), \\
s_{7}= & \frac{-\left\langle\gamma_{x}\right\rangle^{2} \alpha h(1+\alpha h)}{2 h\left(\left\langle\gamma_{x}\right\rangle+\left\langle\gamma_{x}\right\rangle \alpha h+\beta\right)} \\
& +\frac{\left\langle\gamma_{x}\right\rangle(1+\alpha h)\left(\left\langle\gamma_{y}\right\rangle+\left\langle\gamma_{y}\right\rangle \alpha h-\beta\right)}{2 h\left(\left\langle\gamma_{x}\right\rangle+\left\langle\gamma_{x}\right\rangle \alpha h+\beta\right)} \\
& +\frac{\beta\left(\left\langle\gamma_{y} \alpha h-\beta\right\rangle\right)}{2 h\left(\left\langle\gamma_{x}\right\rangle+\left\langle\gamma_{x}\right\rangle \alpha h+\beta\right)} .
\end{aligned}
$$

The fourth steady-state solution is given by

$$
x_{s 4}=0, \quad y_{s 4}=0 .
$$


The fifth steady-state solution is

$$
x_{s 5}=\frac{\alpha}{\beta}, y_{s 5}=0 .
$$

The stability of the steady solutions is determined by the Jacobian matrix:

$$
\mathbf{J}=\left(\begin{array}{cc}
\frac{\left\langle\gamma_{x}\right\rangle y}{\left.1+\gamma_{x}\right\rangle h y}+\alpha-2 \beta x & -\frac{\left\langle\gamma_{x}{ }^{2} h x y\right.}{\left(1+\left\langle\gamma_{x}\right] / h y\right)^{2}}+\frac{\left\langle\gamma_{x}\right\rangle x}{1+\left\langle\gamma_{x}\right\rangle h y} \\
-\frac{\left\langle\gamma_{y}\right)^{2} h x y}{\left(1+\left\langle\gamma_{y}\right\rangle h\right)^{2}}+\frac{\left\langle\gamma_{y}\right\rangle y}{1+\left\langle\gamma_{y}\right\rangle h x} & \frac{\left\langle\gamma_{y}\right\rangle x}{1+\left\langle\gamma_{y}\right\rangle h x}+\alpha-2 \beta y
\end{array}\right) .
$$

The third steady-state solution gives the unstable steady state. For simplicity, we assume $\left\langle\gamma_{x}\right\rangle \cong \gamma$ and $\left\langle\gamma_{y}\right\rangle \cong \gamma$, which give

$$
x_{s 3}=\frac{-r_{1}+r_{2}+\left[\left(r_{1}-r_{2}\right)^{2}-4 r_{3} r_{4}\right]^{1 / 2}}{2 r_{4}},
$$

where

$$
\begin{aligned}
& r_{1}=\gamma^{2}\left(a b+a b \alpha h-a b \alpha h^{2}\right), \\
& r_{2}=\gamma(a \alpha \beta h-b \alpha \beta h)+\beta^{2}, \\
& r_{3}=\gamma\left(\alpha+\alpha^{2} h\right)+\alpha \beta, \\
& r_{4}=-a b \gamma^{2}\left(h \beta+h^{2} \alpha \beta\right)+b \gamma h \beta^{2},
\end{aligned}
$$

and a similar formula for $y_{s 3}$, from which Figs. 11 and 12 are obtained.

\section{APPENDIX B: METHOD TO SYSTEMATICALLY VARY NESTEDNESS}

In order to vary the nestedness of a mutualistic network systematically, we generate a random mutualistic network and rearrange or rewire the interactions in the network until a desired value of nestedness is achieved [32]. Specifically, we start the rewiring process by randomly choosing an edge, say one between species $i$ and $j$. We then randomly select another species $k$. If $i$ is a pollinator, then $j$ and $k$ must be plant species, and vice versa. If species $k$ has more links than species $j$, we connect species $i$ with $k$; Otherwise, we leave the interaction between $i$ and $j$ unchanged. After each successful random rewiring iteration, we calculate the nestedness of the whole mutualistic network defined as [87]

$$
\text { Nest }=\frac{\sum_{i<j}^{S_{X}} D_{i j}^{X}+\sum_{i<j}^{S_{Y}} D_{i j}^{Y}}{S_{X}\left(S_{X}-1\right) / 2+S_{Y}\left(S_{Y}-1\right) / 2},
$$

where $D_{i j}^{X}=d_{i j}^{X} / \min \left(d_{i}^{X}, d_{j}^{X}\right), D_{i j}^{Y}=d_{i j}^{Y} / \min \left(d_{i}^{Y}, d_{j}^{Y}\right), d_{i j}^{X}$ is the number of plant species that both pollinator species $i$ and $j$ interact with (similarly for $d_{i j}^{Y}$, the corresponding quantity for plant species), and $d_{i}^{X}$ and $d_{j}^{X}$ are the total numbers of plant species that pollinator species $i$ and $j$ interact with, respectively (similarly for $d_{i}^{Y}$ and $d_{j}^{Y}$ ). The rewiring process stops when the nestedness of the network has reached the desired value.
[1] R. M. May, Biological populations with nonoverlapping generations: Stable points, stable cycles, and chaos, Science 186, 645 (1974).

[2] J. L. Aron and I. B. Schwartz, Seasonality and period-doubling bifurcations in an epidemic model, J. Theor. Biol. 110, 665 (1984).

[3] A. Hastings, C. L. Hom, S. Ellner, P. Turchin, and H. C. J. Godfray, Chaos in ecology: Is mother nature a strange attractor? Annu. Rev. Ecol. Syst. 24, 1 (1993).

[4] Q. Yao and H. Tong, On prediction and chaos in stochastic systems, Philos. Trans. R. Soc. London A 348, 357 (1994).

[5] J. Ripa, P. Lundberg, and V. Kaitala, A general theory of environmental noise in ecological food webs, Am. Nat. 151, 256 (1998).

[6] S. P. Ellner and P. Turchin, When can noise induce chaos and why does it matter: A critique, Oikos 111, 620 (2005).

[7] Y.-C. Lai and Y.-R. Liu, Noise Promotes Species Diversity in Nature, Phys. Rev. Lett. 94, 038102 (2005).

[8] Y.-C. Lai, Beneficial role of noise in promoting species diversity through stochastic resonance, Phys. Rev. E 72, 042901 (2005).

[9] V. Guttal and C. Jayaprakash, Impact of noise on bistable ecological systems, Ecol. Model. 201, 420 (2007).

[10] S. C. Doney and S. F. Sailley, When an ecological regime shift is really just stochastic noise, Proc. Natl. Acad. Sci. USA 110, 2438 (2013).

[11] O. N. Bjornstad, Nonlinearity and chaos in ecological dynamics revisited, Proc. Natl. Acad. Sci. USA 112, 6252 (2015).

[12] S. M. O'Regan, How noise and coupling influence leading indicators of population extinction in a spatially extended ecological system, J. Biol. Dyn. 12, 211 (2018).
[13] A. Hastings, K. C. Abbott, K. Cuddington, T. Francis, G. Gellner, Y.-C. Lai, A. Morozov, S. Petrovskii, K. Scranton, and M. L. Zeeman, Transient phenomena in ecology, Science 361, eaat6412 (2018).

[14] R. D. Holt and M. A. McPeek, Chaotic population dynamics favors the evolution of dispersal, Am. Nat. 148, 709 (1996).

[15] M. A. Harrison, Y.-C. Lai, and R. D. Holt, A dynamical mechanism for coexistence of dispersing species without trade-offs in spatially extended ecological systems, Phys. Rev. E 63, 051905 (2001).

[16] M. A. Harrison, Y.-C. Lai, and R. D. Holt, Dynamical mechanism for coexistence of dispersing species, J. Theo. Biol. 213, 53 (2001).

[17] R. Benzi, A. Sutera, and A. Vulpiani, The mechanism of stochastic resonance, J. Phys. A 14, L453 (1981).

[18] R. Benzi, G. Parisi, A. Sutera, and A. Vulpiani, A theory of stochastic resonance in climatic-change, J. Appl. Math. 43, 565 (1983).

[19] B. McNamara and K. Wiesenfeld, Theory of stochastic resonance, Phys. Rev. A 39, 4854 (1989).

[20] G. Hu, T. Ditzinger, C. Z. Ning, and H. Haken, Stochastic Resonance without External Periodic Force, Phys. Rev. Lett. 71, 807 (1993).

[21] F. Moss, D. Pierson, and D. O'Gorman, Stochastic resonanceTutorial and update, Int. J. Bif. Chaos 4, 1383 (1994).

[22] P. C. Gailey, A. Neiman, J. J. Collins, and F. Moss, Stochastic Resonance in Ensembles of Bondynamical Elements: The Role of Internal Noise, Phys. Rev. Lett. 79, 4701 (1997).

[23] L. Gammaitoni, P. Hänggi, P. Jung, and F. Marchesoni, Stochastic resonance, Rev. Mod. Phys. 70, 223 (1998). 
[24] D. Sigeti and W. Horsthemke, Pseudo-regular oscillations induced by external noise, J. Stat. Phys. 54, 1217 (1989).

[25] A. S. Pikovsky and J. Kurths, Coherence Resonance in a NoiseDriven Excitable System, Phys. Rev. Lett. 78, 775 (1997).

[26] Z. Liu and Y.-C. Lai, Coherence Resonance in Coupled Chaotic Oscillators, Phys. Rev. Lett. 86, 4737 (2001).

[27] Y.-C. Lai and Z. Liu, Noise-enhanced temporal regularity in coupled chaotic oscillators, Phys. Rev. E 64, 066202 (2001).

[28] P. Hessari, Y. Do, Y.-C. Lai, J. Chae, C. W. Park, and G. W. Lee, Regularization of chaos by noise in electrically driven nanowire systems, Phys. Rev. B 89, 134304 (2014).

[29] J. Bascompte, P. Jordano, C. J. Melián, and J. M. Olesen, The nested assembly of plant-animal mutualistic networks, Proc. Natl. Acad. Sci. USA 100, 9383 (2003).

[30] P. R. Guimaraes, P. Jordano, and J. N. Thompson, Evolution and coevolution in mutualistic networks, Ecol. Lett. 14, 877 (2011).

[31] S. L. Nuismer, P. Jordano, and J. Bascompte, Coevolution and the architecture of mutualistic networks, Evolution 67, 338 (2013).

[32] J. J. Lever, E. H. Nes, M. Scheffer, and J. Bascompte, The sudden collapse of pollinator communities, Ecol. Lett. 17, 350 (2014).

[33] R. P. Rohr, S. Saavedra, and J. Bascompte, On the structural stability of mutualistic systems, Science 345, 1253497 (2014).

[34] V. Dakos and J. Bascompte, Critical slowing down as early warning for the onset of collapse in mutualistic communities, Proc. Natl. Acad. Sci. USA 111, 17546 (2014).

[35] P. R. Guimaraes, M. M. Pires, P. Jordano, J. Bascompte, and J. N. Thompson, Indirect effects drive coevolution in mutualistic networks, Nature (London) 550, 511 (2017).

[36] J. Jiang, Z.-G. Huang, T. P. Seager, W. Lin, C. Grebogi, A. Hastings, and Y.-C. Lai, Predicting tipping points in mutualistic networks through dimension reduction, Proc. Natl. Acad. Sci. USA 115, E639 (2018).

[37] T. Ohgushi, O. Schmitz, and R. D. Holt, Trait-Mediated Indirect Interactions: Ecological and Evolutionary Perspectives (Cambridge University Press, Cambridge, 2012).

[38] P. P. A. Staniczenko, J. C. Kopp, and S. Allesina, The ghost of nestedness in ecological networks, Nat. Commun. 4, 1391 (2013).

[39] J. Bronstein, Mutualism (Oxford University Press, Oxford, 2015).

[40] M. Scheffer, Ecology of Shallow Lakes (Springer Science \& Business Media, New York, 2004).

[41] M. Scheffer, J. Bascompte, W. A. Brock, V. Brovkin, S. R. Carpenter, V. Dakos, H. Held, E. H. Van Nes, M. Rietkerk, and G. Sugihara, Early-warning signals for critical transitions, Nature (London) 461, 53 (2009).

[42] M. Scheffer, Complex systems: Foreseeing tipping points, Nature (London) 467, 411 (2010).

[43] D. B. Wysham and A. Hastings, Regime shifts in ecological systems can occur with no warning, Ecol. Lett. 13, 464 (2010).

[44] J. M. Drake and B. D. Griffen, Early warning signals of extinction in deteriorating environments, Nature (London) 467, 456 (2010).

[45] C. Boettiger and A. Hastings, Quantifying limits to detection of early warning for critical transitions, J. R. Soc. Interface 9, 2527 (2012).

[46] L. Dai, D. Vorselen, K. S. Korolev, and J. Gore, Generic indicators for loss of resilience before a tipping point leading to population collapse, Science 336, 1175 (2012).

[47] P. Ashwin, S. Wieczorek, R. Vitolo, and P. Cox, Tipping points in open systems: Bifurcation, noise-induced and rate-dependent examples in the climate system, Philos. Trans. R. Soc. London A 370, 1166 (2012).

[48] T. M. Lenton, V. N. Livina, V. Dakos, E. H. van Nes, and M. Scheffer, Early warning of climate tipping points from critical slowing down: Comparing methods to improve robustness, Philos. Trans. R. Soc. London A 370, 1185 (2012).

[49] A. D. Barnosky, E. A. Hadly, J. Bascompte, E. L. B. J. H. Brown, M. Fortelius, W. M. Getz, J. Harte, A. Hastings, P. A. Marquet, N. D. Martinez, A. Mooers, P. Roopnarine, G. Vermeij, J. W. Williams, R. Gillespie, J. Kitzes, C. Marshall, N. Matzke, D. P. Mindell, E. Revilla, and A. B. Smith, Approaching a state shift in earth's biosphere, Nature (London) 486, 52 (2012).

[50] C. Boettiger and A. Hastings, Tipping points: From patterns to predictions, Nature (London) 493, 157 (2013).

[51] J. M. Tylianakis and C. Coux, Tipping points in ecological networks, Trends Plant. Sci. 19, 281 (2014).

[52] T. S. Lontzek, Y.-Y. Cai, K. L. Judd, and T. M. Lenton, Stochastic integrated assessment of climate tipping points indicates the need for strict climate policy, Nat. Clim. Change 5, 441 (2015).

[53] S. Gualdia, M. Tarziaa, F. Zamponic, and J.-P. Bouchaudd, Tipping points in macroeconomic agent-based models, J. Econ. Dyn. Contr. 50, 29 (2015).

[54] J. Jiang, A. Hastings, and Y.-C. Lai, Harnessing tipping points in complex ecological networks, J. R. Soc. Interface 16, 20190345 (2019).

[55] M. Gladwell, The Tipping Point: How Little Things Can Make a Big Difference (Little, Brown and Company, New York, 2000).

[56] C. S. Holling, Some characteristics of simple types of predation and parasitism, Can. Entomol. 91, 385 (1959).

[57] C. S. Holling, Resilience and stability of ecological systems, Annu. Rev. Ecol. Syst. 4, 1 (1973).

[58] C. Van den Broeck, J. M. R. Parrondo, R. Toral, and R. Kawai, Nonequilibrium phase transitions induced by multiplicative noise, Phys. Rev. E 55, 4084 (1997).

[59] J. Ollerton, Pollinator diversity: Distribution, ecological function, and conservation, Rev. Ecol. Evol. Syst. 48, 353 (2017).

[60] C. M. Herrera, Components of pollinator quality: Comparative analysis of a diverse insect assemblage, Oikos 50, 79 (1987).

[61] M. C. Rodríguez-Rodríguez, P. Jordano, and A. Valido, Quantity and quality components of effectiveness in insular pollinator assemblages, Oecologia 173, 179 (2013).

[62] G. Ballantyne, K. C. R. Baldock, and P. G. Willmer, Constructing more informative plant-pollinator networks: Visitation and pollen deposition networks in a heathland plant community, Proc. Roy. Soc. B 282, 20151130 (2015).

[63] P. G. Willmer and K. Finlayson, Big bees do a better job: Intraspecific size variation influences pollination effectiveness, J. Pollination Ecol. 14, 244 (2014).

[64] Available from the Web of Life database, http://www.web-oflife.es.

[65] L. Dicks, S. Corbet, and R. Pywell, Compartmentalization in plant-insect flower visitor webs, J. Anim. Ecol. 71, 32 (2002).

[66] Y. L. Dupont, D. M. Hansen, and J. M. Olesen, Structure of a plant-flower-visitor network in the high-altitude sub-alpine desert of Tenerife, Canary Islands, Ecography 26, 301 (2003). 
[67] A. F. Motten, Pollination ecology of the spring wildflower community in the deciduous forests of Piedmont North Carolina, Ph.D. thesis, Duke University, 1982.

[68] A. C. Montero, The ecology of three pollinator network, Master's thesis, Aarhus University, Aarhus, Denmark, 2005.

[69] S. Jonhson, V. Dominguez-Garcia, and M. A. Munoz, Factors determining nestedness in complex networks, PLoS ONE 8, e74025 (2013).

[70] C. Payrató-Borràs, L. Hernández, and Y. Moreno, Breaking the Spell of Nestedness: The Entropic Origin of Nestedness in Mutualistic Systems, Phys. Rev. X 9, 031024 (2019).

[71] U. Feudel, C. Grebogi, B. R. Hunt, and J. A. Yorke, Map with more than 100 coexisting low-period periodic attractors, Phys. Rev. E 54, 71 (1996).

[72] U. Feudel and C. Grebogi, Multistability and the control of complexity, Chaos 7, 597 (1997).

[73] S. Kraut, U. Feudel, and C. Grebogi, Preference of attractors in noisy multistable systems, Phys. Rev. E 59, 5253 (1999).

[74] S. Kraut and U. Feudel, Multistability, noise, and attractor hopping: The crucial role of chaotic saddles, Phys. Rev. E 66, 015207(R) (2002).

[75] U. Feudel and C. Grebogi, Why are Chaotic Attractors Rare in Multistable Systems? Phys. Rev. Lett. 91, 134102 (2003).

[76] C. N. Ngonghala, U. Feudel, and K. Showalter, Extreme multistability in a chemical model system, Phys. Rev. E 83, 056206 (2011).

[77] M. S. Patel, U. Patel, A. Sen, G. C. Sethia, C. Hens, S. K. Dana, U. Feudel, K. Showalter, C. N. Ngonghala, and R. E. Amritkar, Experimental observation of extreme multistability in an electronic system of two coupled Rössler oscillators, Phys. Rev. E 89, 022918 (2014).

[78] A. N. Pisarchik and U. Feudel, Control of multistability, Phys. Rep. 540, 167 (2014).

[79] Y.-C. Lai and C. Grebogi, Quasiperiodicity and suppression of multistability in nonlinear dynamical systems, Euro. Phys. J. Spec. Top. 226, 1703 (2017).

[80] S. W. McDonald, C. Grebogi, E. Ott, and J. A. Yorke, Fractal basin boundaries, Physica D (Amsterdam) 17, 125 (1985).

[81] Y.-C. Lai and T. Tél, Transient Chaos-Complex Dynamics on Finite Time Scales (Springer, New York, 2011).

[82] S.-H. Chang, P. C. Cosman, and L. B. Milstein, Chernoff-type bounds for the Gaussian error function, IEEE Trans. Commun. 59, 2939 (2011).

[83] M. B. Bonsall and A. Hastings, Demographic and environmental stochasticity in predator-prey metapopulation dynamics, J. Animal Ecol. 73, 1043 (2004).

[84] P. V. Martín, J. A. Bonachela, S. A. Levin, and M. A. Muñoz, Eluding catastrophic shifts, Proc. Natl. Acad. Sci. USA 112, E1828 (2015)

[85] G. W. A. Constable, T. Rogers, A. J. McKane, and C. E. Tarnita, Demographic noise can reverse the direction of deterministic selection, Proc. Natl. Acad. Sci. USA 113, E4745 (2016).

[86] H. Weissmann, N. M. Shnerb, and D. A. Kessler, Simulation of spatial systems with demographic noise, Phys. Rev. E 98, 022131 (2018).

[87] M. Almeida-Neto, P. Guimaraes, P. R. Guimaraes, Jr., R. D. Loyola, and W. Ulrich, A consistent metric for nestedness analysis in ecological systems: Reconciling concept and measurement, Oikos 117, 1227 (2008). 